\title{
Case Report \\ Heart Failure with Transient Left Bundle Branch Block in the Setting of Left Coronary Fistula
}

\author{
Stephen P. Juraschek, Lara C. Kovell, Ryan E. Childers, Grant V. Chow, and Glenn A. Hirsch \\ Department of Medicine, Johns Hopkins Bayview Medical Center, Johns Hopkins University School of Medicine, \\ 4940 Eastern Avenue, Baltimore, MD 21224, USA \\ Correspondence should be addressed to Stephen P. Juraschek, spj@jhmi.edu
}

Received 25 November 2010; Accepted 11 January 2011

Academic Editor: Hendrik T. Tevaearai

Copyright $\odot 2011$ Stephen P. Juraschek et al. This is an open access article distributed under the Creative Commons Attribution License, which permits unrestricted use, distribution, and reproduction in any medium, provided the original work is properly cited.

Coronary arterial fistulas are rare communications between vessels or chambers of the heart. Although cardiac symptoms associated with fistulas are well described, fistulas are seldom considered in the differential diagnosis of acute myocardial ischemia. We describe the case of a 64-year-old man who presented with left shoulder pain, signs of heart failure, and a new left bundle branch block (LBBB). Cardiac catheterization revealed a small left anterior descending (LAD)-to-pulmonary artery (PA) fistula. Diuresis led to subjective improvement of the patient's symptoms and within several days the LBBB resolved. We hypothesize that the coronary fistula in this patient contributed to transient ischemia of the LAD territory through a coronary steal mechanism. We elected to observe rather than repair the fistula, as his symptoms and ECG changes resolved with treatment of his heart failure.

\section{Introduction}

Coronary arterial fistulas are rare cardiac anomalies that create new pathways of blood flow between coronary vessels and thoracic vasculature or chambers of the heart [1]. Although generally asymptomatic, patients can develop complications of thrombosis, congestive heart failure, rupture, endocarditis, and arrhythmias [2]. Here, we describe a patient with acute decompensated heart failure and transient left bundle branch block (LBBB), found to have an underlying left anterior descending (LAD)-to-pulmonary artery (PA) fistula. This case illustrates the potential of coronary fistulas to induce a clinical presentation of cardiac ischemia via a coronary steal mechanism.

\section{Case Report}

A 64-year-old man with a history of congestive heart failure and stage IV chronic kidney disease secondary to uncontrolled hypertension and diabetes mellitus presented with three days of orthopnea and one night of intermittent left shoulder pain radiating to the back.
Physical examination revealed a heart rate of 99 beats per minute and blood pressure of $149 / 71 \mathrm{~mm} \mathrm{Hg}$ with an oxygen saturation of $94 \%$ on 4 liters of oxygen. Bilateral rales were present in the lower and middle lung fields. Cardiac auscultation revealed distant heart sounds and a regular rate and rhythm without murmur. Jugular venous pressure was elevated to $10 \mathrm{~cm} \mathrm{H}_{2} \mathrm{O}$. Lower extremity pitting edema $(2+)$ was present. Laboratory analyses were significant for a hematocrit of $24 \%$ and creatinine of $3.8 \mathrm{mg} / \mathrm{dL}$. Cardiac enzymes were normal. Twelve-lead electrocardiography (ECG) revealed sinus rhythm at 94 beats per minute and a new LBBB.

An acute myocardial infarction was suspected and the patient was taken for emergent cardiac catheterization, which showed stenosis of up to $40 \%$ of the mid-LAD coronary artery and mild disease in other vessels. In addition, a fistula was identified connecting the LAD to the distal main PA (Figures 1(a) and 1(b)). The direction of blood flow was from the coronary vessel into the pulmonary circulation. No acute intervention was performed at the time of cardiac catheterization. Transthoracic echocardiography demonstrated moderate global systolic dysfunction 


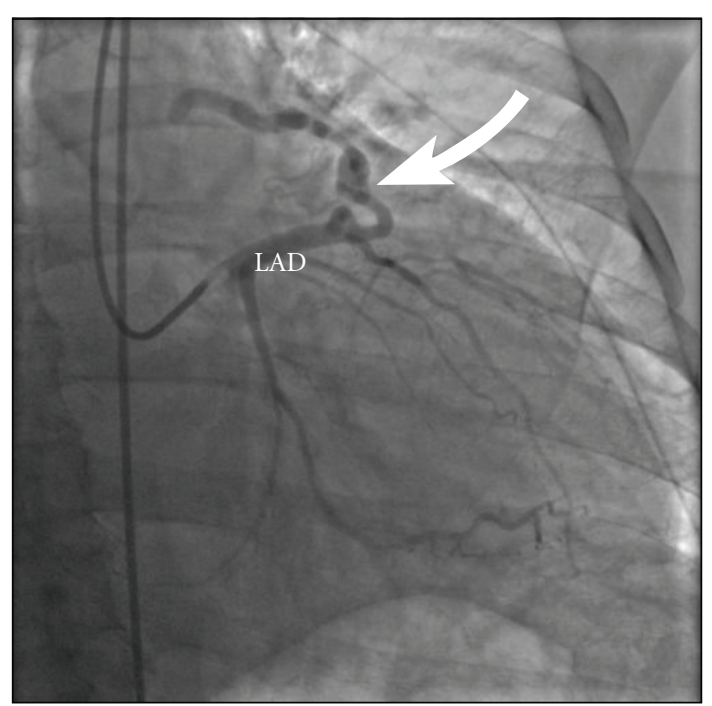

(a)

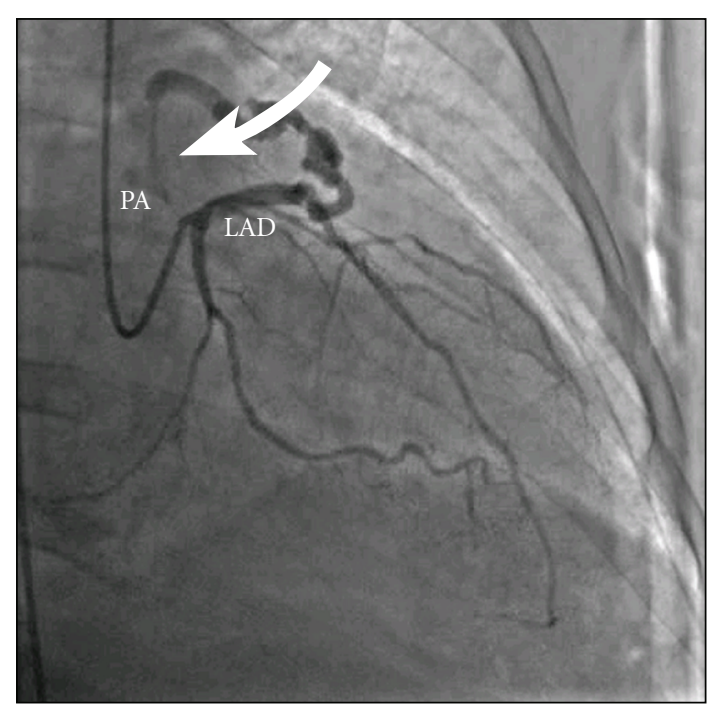

(b)

FIGURE 1: Right anterior oblique cranial views during coronary angiography revealed a fistula (arrow, a) arising from the left anterior descending (LAD) artery, which was then found to empty into the pulmonary artery (PA) via a small branch (arrow, b).

with a left ventricular ejection fraction of $40 \%$ as well as a moderate-sized pericardial effusion without echocardiographic evidence of increased intrapericardial pressure. The patient was diuresed with intravenous furosemide with gradual symptomatic and objective improvement.

A regadenoson nuclear perfusion stress imaging study performed after 4 days of diuresis and a blood transfusion was negative for inducible ischemia. Furthermore, an ECG on the day of the stress imaging study showed resolution of the LBBB, and one week later the patient's ECG showed recovery to near normal left ventricular ejection fraction and decrease in size of the pericardial effusion.

We hypothesized that the small LAD-PA fistula was not the sole cause of heart failure symptoms but was a contributor to the patient's clinical presentation and ECG findings. The small fistula in this case "steals" from the LADsupplied myocardium, including the left bundle. By itself, the fistula was not sufficient to manifest clinically. However, in the setting of increased oxygen demand and decreased oxygen supply during the heart failure exacerbation, this coronary steal likely decreased the total oxygen supply in the LAD territory enough to cause additional transient ischemia and LBBB.

The patient was discharged after 7 days. Since he was previously asymptomatic without objective evidence of ischemia in the absence of myocardial oxygen supply and demand mismatch, we elected to continue close observation rather than closure of the fistula.

\section{Discussion}

Coronary arterial fistulas are rare communications between coronary vasculature and thoracic vessels or cardiac chambers [1]. The most frequent sites of drainage include the ventricles, pulmonary arteries, the coronary sinus, the superior vena cava, or the pulmonary veins [1]. Of the different types of fistulas, $42 \%$ originate from the left coronary tree and $17 \%$ drain into the pulmonary artery [3]. Fistulas are generally congenital in origin, but they can also arise from cardiac trauma, chest irradiation, cardiac surgery, coronary angioplasty, and endomyocardial biopsy [1].

While fistulas are usually asymptomatic, their natural history can be variable. Adult patients who develop symptoms often do so in the 5 th or 6 th decade $[1,2]$. Common clinical presentations include dyspnea, congestive heart failure, angina, aneurysm, or myocardial infarction [4]. Whether or not a patient will develop symptoms is determined by the degree of fistula-induced volume overload, as well as the severity of left-to-right shunting secondary to fistula size and location [1].

Coronary angiography is the primary diagnostic tool for defining fistula anatomy [2]. Transesophageal echocardiogram may be useful in delineating the origin, course, and drainage of a fistula [5]. Closure rarely occurs spontaneously in adults. Therefore, in the presence of large shunts or even in asymptomatic patients, surgical or transcatheter ligation may be considered to prevent long-term sequelae such as steal, spontaneous rupture, heart failure, or myocardial ischemia $[6,7]$. Transcatheter closure is first-line therapy in suitable anatomic cases due to lower cost, shorter recovery time, and reduced hospital stay. This avoids thoracotomy and cardiopulmonary bypass, which could accompany a surgical approach [8].

This case has several limitations. Although coronary steal may have contributed to ischemia of the left bundle as evidenced by subsequent ECG findings, we cannot exclude a rate-related bundle branch block. However, this is less likely since an ECG tracing 5 months earlier, at the same heart rate, revealed a normal QRS duration as did 
subsequent ECG tracings at similar heart rates. A right heart catheterization was not performed to assess filling pressures or to calculate a shunt fraction. Even without direct measures of these pressures, it was still evident that the patient was in congestive heart failure by history and physical exam findings. He improved significantly with diuresis, suggesting elevated filling pressures also contributed to the supplydemand mismatch. The left ventricular systolic function may have also appeared decreased at presentation because of the septal motion abnormality from the LBBB.

\section{Conclusions}

Our patient, who presented with orthopnea, shoulder pain, and a new LBBB, illustrates that even a small coronary fistula may "steal" enough blood flow to mimic the symptoms of an acute myocardial infarction in the setting of a transient oxygen supply-demand mismatch. A coronary fistula, therefore, may not be the sole culprit lesion or an innocent bystander, but an accomplice in causing signs and symptoms of acute heart failure.

\section{Disclosures and Financial Support}

The authors have no disclosures or financial conflicts of interest relevant to this paper to report. All authors contributed to the writing, editing, and content of this manuscript.

\section{References}

[1] S. Balanescu, G. Sangiorgi, S. Castelvecchio, M. Medda, and L. Inglese, "Coronary artery fistulas: clinical consequences and methods of closure. A literature review," Italian Heart Journal, vol. 2, no. 9, pp. 669-676, 2001.

[2] S. A. Qureshi, "Coronary arterial fistulas," Orphanet Journal of Rare Diseases, vol. 1, no. 51, pp. 1-6, 2006.

[3] D. C. Levin, K. E. Fellows, and H. L. Abrams, "Hemodynamically significant primary anomalies of the coronary arteries. Angiographic aspects," Circulation, vol. 58, no. 1, pp. 25-34, 1978.

[4] R. M. Gowda, B. C. Vasavada, and I. A. Khan, "Coronary artery fistulas: clinical and therapeutic considerations," International Journal of Cardiology, vol. 107, no. 1, pp. 7-10, 2006.

[5] L. Olivotti, S. Moshiri, G. Santoro, A. Nicolino, and F. Chiarella, "Percutaneous closure of a giant coronary arteriovenous fistula using free embolization coils in an adult patient," Journal of Cardiovascular Medicine, vol. 9, no. 7, pp. 733-736, 2008.

[6] T. Tirilomis, I. Aleksic, T. Busch, D. Zenker, W. Ruschewski, and H. Dalichau, "Congenital coronary artery fistulas in adults: surgical treatment and outcome," International Journal of Cardiology, vol. 98, no. 1, pp. 57-59, 2005.

[7] Y. Ata, T. Turk, M. Bicer, M. Yalcin, F. Ata, and S. Yavuz, "Coronary arteriovenous fistulas in the adults: natural history and management strategies," Journal of Cardiothoracic Surgery, vol. 4, no. 1, p. 62, 2009.

[8] X. Y. Zhu, D. Z. Zhang, X. M. Han et al., “Transcatheter closure of congenital coronary artery fistulae: immediate and long-term follow-up results," Clinical Cardiology, vol. 32, no. 9, pp. 506512, 2009. 


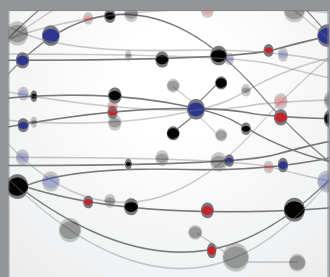

The Scientific World Journal
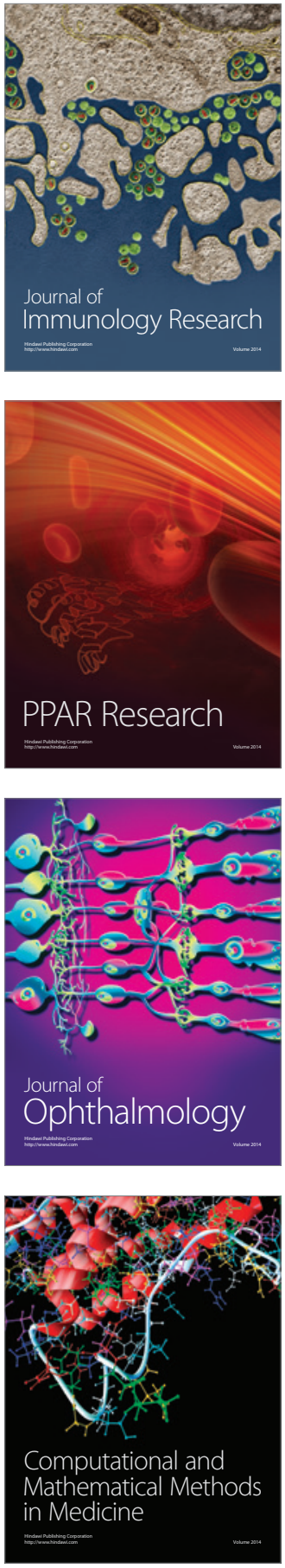

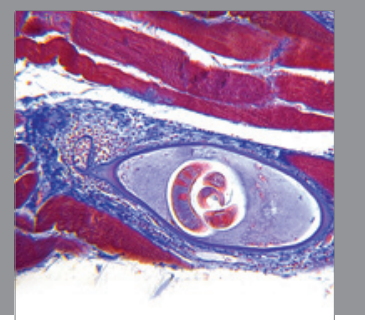

Gastroenterology

Research and Practice
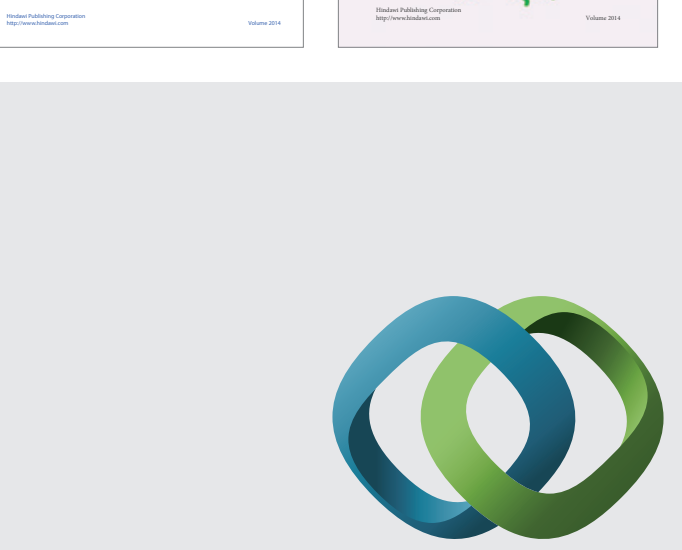

\section{Hindawi}

Submit your manuscripts at

http://www.hindawi.com
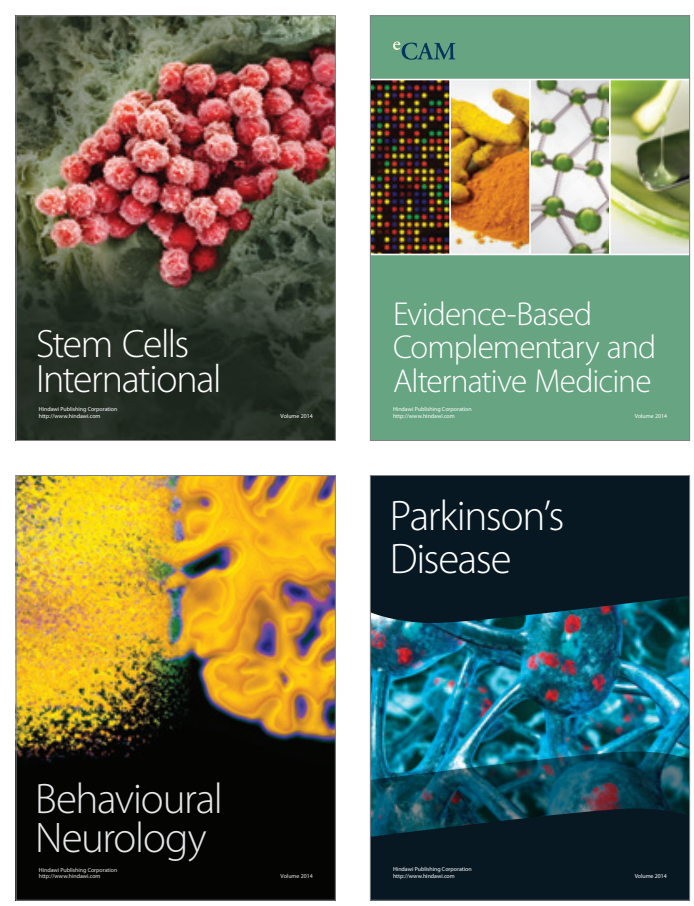

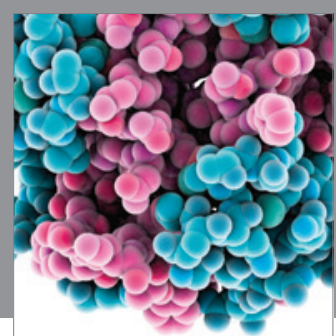

Journal of
Diabetes Research

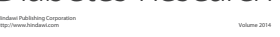

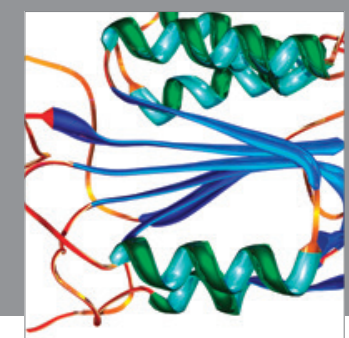

Disease Markers
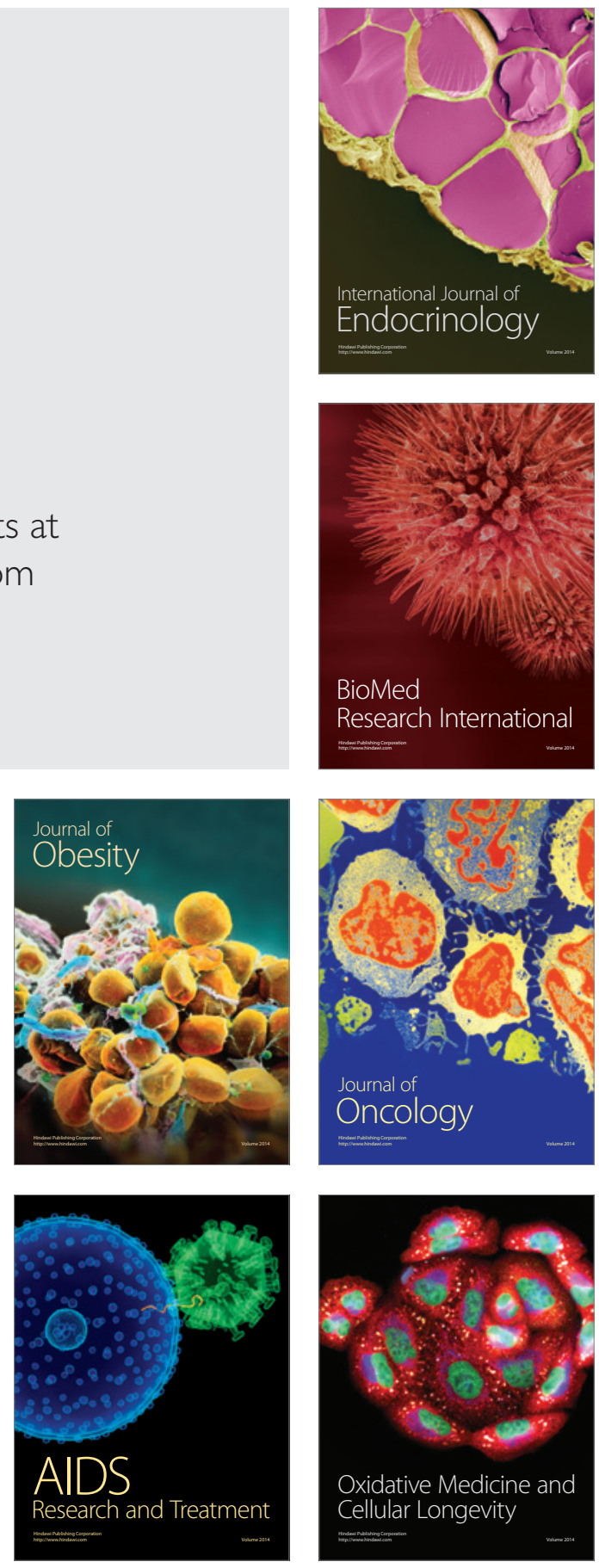